Article

\title{
Impact of Smoking-Related Chronic Obstruction Pulmonary Disease on Mortality of Invasive Ductal Carcinoma Patients Receiving Standard Treatments: Propensity Score-Matched, Nationwide, Population-Based Cohort Study
}

Jia-Qiang Zhang ${ }^{1,2,+}$, Tsai-Mu Cheng ${ }^{3,+}{ }^{+}$, Wei-Chun Lin ${ }^{4}$, Kuo-Chin Chiu ${ }^{4, *}$ and Szu-Yuan Wu ${ }^{1,2,5,6,7,8,9,10, *}$

1 Department of Anesthesiology and Perioperative Medicine, Henan Provincial People's Hospital, People's Hospital of Zhengzhou University, Zhengzhou 450052, China; jqzhang@henu.edu.cn

2 Centers for Regional Anesthesia and Pain Medicine, Wan Fang Hospital, Taipei Medical University, Taipei 110, Taiwan

3 The Ph.D. Program for Translational Medicine, College of Medical Science and Technology, Taipei Medical University, Taipei 110, Taiwan; tmcheng@tmu.edu.tw

4 Division of Chest Medicine, Department of Internal Medicine, Lo-Hsu Medical Foundation, Lotung Poh-Ai Hospital, Yilan 256, Taiwan; 973002@mail.pohai.org.tw

5 Department of Food Nutrition and Health Biotechnology, College of Medical and Health Science, Asia University, Taichung 413, Taiwan

check for updates

Citation: Zhang, J.-Q.; Cheng, T.-M.; Lin, W.-C.; Chiu, K.-C.; Wu, S.-Y. Impact of Smoking-Related Chronic Obstruction Pulmonary Disease on Mortality of Invasive Ductal Carcinoma Patients Receiving Standard Treatments: Propensity Score-Matched, Nationwide, Population-Based Cohort Study. Cancers 2021, 13, 3654. https://doi.org/10.3390/ cancers13153654

Academic Editors: Tommaso Susini and Nicoletta Biglia

Received: 8 July 2021

Accepted: 19 July 2021

Published: 21 July 2021

Publisher's Note: MDPI stays neutral with regard to jurisdictional claims in published maps and institutional affiliations.

Copyright: (c) 2021 by the authors. Licensee MDPI, Basel, Switzerland. This article is an open access article distributed under the terms and conditions of the Creative Commons Attribution (CC BY) license (https:// creativecommons.org/licenses/by/ $4.0 /)$.
6 Big Data Center, Lo-Hsu Medical Foundation, Lotung Poh-Ai Hospital, Yilan 256, Taiwan

7 Division of Radiation Oncology, Lo-Hsu Medical Foundation, Lotung Poh-Ai Hospital, Yilan 256, Taiwan

8 Department of Healthcare Administration, College of Medical and Health Science, Asia University, Taichung 413, Taiwan

9 Cancer Center, Lo-Hsu Medical Foundation, Lotung Poh-Ai Hospital, Yilan 256, Taiwan

10 Graduate Institute of Business Administration, Fu Jen Catholic University, Taipei 242062, Taiwan

* Correspondence: 979010@mail.pohai.org.tw (K.-C.C.); szuyuan@tmu.edu.tw (S.-Y.W.)

$\dagger \quad$ The authors contributed equally to this study.

Simple Summary: This study is the first to estimate the impact of smoking-related chronic obstructive pulmonary disease (COPD) on invasive ductal carcinoma (IDC) patients receiving standard treatments. Smoking-related COPD was not a significant independent risk factor for all-cause mortality in women with stage I-III IDC receiving standard treatments. The frequency of hospitalization for COPD with at least one acute exacerbation within one year before breast surgery was highly associated with high mortality for women with IDC receiving standard treatments.

Abstract: Purpose: the survival effect of smoking-related chronic obstructive pulmonary disease (COPD) and COPD with acute exacerbation (COPDAE) is unclear for patients with invasive ductal carcinoma (IDC) receiving standard treatments. Methods: we recruited women with clinical stage I-III IDC from the Taiwan Cancer Registry Database who had received standard treatments between 1 January 2009 and 31 December 2018. The time-dependent Cox proportional hazards model was used to analyze all-cause mortality. To reduce the effects of potential confounders when all-cause mortality between Groups 1 and 2 were compared, 1:2 propensity score matching (PSM) was performed. We categorized the patients into two groups based on COPD status to compare overall survival outcomes: Group 1 (current smokers with COPD) and Group 2 (nonsmokers without COPD group). Results: PSM yielded 2319 patients with stage I-III IDC (773 and 1546 in Groups 1 and 2, respectively) eligible for further analysis. In the multivariate time-dependent Cox regression analyses, the adjusted hazard ratio (aHR; 95\% confidence interval (CI)) of all-cause mortality for Group 1 compared with Group 2 was $1.04(0.83-1.22)$. The aHRs (95\% CIs) of all-cause mortality for $\geq 1$ hospitalization for COPDAE within one year before breast surgery was 1.51 (1.18-2.36) compared with no COPDAE. Conclusion: smoking-related COPD was not a significant independent risk factor for all-cause mortality in women with stage I-III IDC receiving standard treatments. Being hospitalized at least once for COPDAE within one year before breast surgery is highly associated with high mortality for women with IDC receiving standard treatments. The severity of smoking-related COPD before treatments 
for breast cancer might be an important prognostic factor of survival. Thus, the information of the severity of COPD before treatment for breast cancer might be valuable for increasing the survival rate in treatment of breast cancer, especially in the prevention of progress from COPD to COPDAE.

Keywords: breast intraductal carcinoma; COPD; COPDAE; cigarette smoking; survival

\section{Introduction}

Smoking-related chronic obstructive pulmonary disease (COPD) and COPD with acute exacerbation (COPDAE) may indicate severe lung inflammation or poor heart function, and may be a surrogate marker of pulmonary or cardiac function [1-4]. COPD or COPDAE may trigger major adverse cardiac events (MACE) [5]. The risk of MACE increases substantially following COPDAE [5]. Prevention of such MACE is a critical goal in COPD management to avoid COPDAE [5]. Many studies have indicated an increased risk of breast cancer in smokers [6-10]. The relationship between cigarette smoking and breast cancer is complicated.

No study has analyzed the severity of COPD and survival outcomes for women with invasive ductal carcinoma (IDC) receiving curative standard treatments (breast surgery followed by adjuvant chemotherapy, anti-human epidermal growth factor receptor 2 [HER2] tyrosine kinase inhibitors, hormone therapy, or adjuvant irradiation according to the National Comprehensive Cancer Network [NCCN] guidelines [11]), although many studies have concluded that having previously experienced MACE causes poor survival in women with breast cancer undergoing breast surgery followed by systemic chemotherapy or adjuvant radiotherapy [12-18]. The cardiotoxicity or lung injury of chemotherapy and radiotherapy can be more severe in women who have experienced MACE or have underlying comorbidities such as COPD [12-20]; having had MACE or having COPD or COPDAE may be risk factors for all-cause mortality for women with breast cancer receiving standard treatments.

Therefore, we assessed whether the severity of smoking-related COPD (COPD, or hospitalization for COPDAE before standard treatments for patients with breast cancer) is an independent prognostic factor of overall survival (OS) in patients with IDC undergoing breast surgery followed by adjuvant treatments based on NCCN guidelines [11]. The severity of COPD before breast cancer treatment may be an important prognostic factor for survival. Therefore, understanding the severity of COPD before breast cancer treatment may be of great significance to improve the survival rate of breast cancer treatment, especially to prevent COPD from progressing to COPDAE.

\section{Patients and Methods}

\subsection{Study Population}

We enrolled patients from the Taiwan Cancer Registry Database (TCRD) with a diagnosis of American Joint Committee on Cancer (AJCC) clinical stage I-III breast IDC between 1 January 2009 and 31 December 2018. The index date was the date of breast surgery, and the follow-up duration was from the index date to 31 December 2019. The TCRD contains detailed cancer-related information of patients, including the stage, cigarette smoking habit, treatment modalities, pathologic data, irradiation doses, hormone receptor (HR) status, HER2 status, radiotherapy, and chemotherapy regimens used [21-26]. The study protocols were reviewed and approved by the Institutional Review Board of the Tzu-Chi Medical Foundation (IRB109-015-B).

\subsection{Inclusion and Exclusion Criteria}

The diagnoses of the enrolled patients were confirmed after reviewing their pathological data, and the women with newly diagnosed IDC were confirmed to have no other cancers or distant metastases. The women were included if they had received an IDC diagnosis, were 20 years old or older, and had clinical stage I-III (AJCC, 8th edition) without metastasis. Patients 
were excluded if they had a history of cancer before the IDC diagnosis date, unknown pathologic types, missing sex data, unclear staging, and non-IDC histology. In addition, patients with nonstandard adjuvant breast radiotherapy (in contrast with standard adjuvant radiotherapy, consisting of irradiation to both the chest wall/whole breast and regional nodes with a minimum of $50 \mathrm{~Gy}$ ), neoadjuvant chemotherapy, unclear differentiation of tumor grade, missing HR status, missing HER2 status, or unclear staging were excluded. Adjuvant treatments such as adjuvant radiotherapy, adjuvant chemotherapy, hormone therapy, or target therapy were allowed based on NCCN guidelines in Taiwan [11]. We also excluded patients with unclear surgical procedures, ill-defined nodal surgery, unclear Charlson comorbidity index (CCI), or unclear differentiation from our cohort. HR positivity was defined as $\geq 1 \%$ of tumor cells demonstrating positive nuclear staining through immunohistochemistry [27], and HER2 positivity was defined as an immunohistochemistry score of $3+$ or a fluorescence in situ hybridization ratio of $\geq 2[28,29]$.

After applying the inclusion and exclusion criteria, we enrolled 2319 women with AJCC clinical stage I-III IDC who had received breast surgery and a sentinel lymph node biopsy (SLNB) or axillary lymph node dissection (ALND), and divided them into two groups based on their smoking-related COPD status to compare all-cause mortality: Group 1 (current smokers with smoking-related COPD before breast surgery) and Group 2 (nonsmokers without COPD before breast surgery). We also estimated the survival outcome of the severity of smoking-related COPD (frequency of hospitalization for COPDAE with 0 or $\geq 1$ hospitalizations within one year before the index date) and patients with stage I-III IDC undergoing breast surgery. Breast surgery including partial (breast-conserving surgery) and total mastectomy were included in our study. Breast-conserving therapy refers to breast-conserving surgery (BCS; i.e., lumpectomy) typically followed by moderatedose radiation therapy (RT) to eradicate any microscopic residual disease. The incidence of comorbidities was scored using the CCI $[30,31]$. MACE refer to a set of comorbidities frequently used in cardiovascular research $[32,33]$ and, herein, consist of a composite of nonfatal stroke, nonfatal myocardial infarction, cardiovascular event, and admission for heart failure [34-36]. MACE, hypertension, diabetes, COPD, hyperlipidemia, and chronic kidney disease (CKD) were excluded from the CCI scores to prevent repetitive adjustment in multivariate analysis. Only comorbidities observed within 12 months before the index date were included; they were coded and classified according to the International Classification of Diseases, 10th Revision, Clinical Modification (ICD-10-CM) codes at the first admission, or after more than two repetitions of a code were issued at outpatient department visits.

Current smokers were recorded by the national professional cancer registrar in the TCRD, which means an adult who has smoked 100 cigarettes in his or her lifetime and who currently smokes cigarettes. The number of current smokers were recorded by these national professional cancer registrars, certified by the Taiwan Cancer Registry. There is no record for abstinence, because current smokers were defined as a patient with breast IDC who currently smokes cigarettes at the index date. Non-smokers were recorded by these national professional cancer registrars in the TCRD, which means an adult who has never smoked cigarettes in his or her lifetime. The non-smokers were recorded by these national professional cancer registrars, certified by the Taiwan Cancer Registry. COPD group were identified as observed within 12 months before the index date; they were used as the main diagnosis code according to ICD-10-CM codes for the first admission, or the main diagnosis code for the two outpatient visits would be classified in the COPD group. Hospitalization of COPDAE defined within 12 months before the index date were included; they were coded and classified according to the ICD-10-CM codes at the first admission.

\subsection{Propensity Score Matching and Covariates}

To reduce the effects of potential confounders when all-cause mortality between Groups 1 and 2 were compared, 1:2 propensity score matching (PSM) was performed with a caliper of 0.2 for the following variables: age, menopausal status, CCI score, differentiation, AJCC clinical stage, adjuvant chemotherapy, adjuvant radiotherapy, HR status, Her-2 status, nodal 
surgery, types of breast surgery, history of MACE, hypertension, diabetes, hyperlipidemia, alcohol use, drug abuse, and CKD [37]. There were no eligible patients that could not be matched in our study. A time-dependent Cox regression model was only utilized for HRs related to time-dependent variables, namely treatments (chemotherapy and radiotherapy). A Cox regression model was used to regress all-cause mortality on different COPD statuses, with a robust sandwich estimator used to account for clustering within matched sets [38]. Multivariate time-dependent Cox regression analyses were performed to calculate hazard ratios to determine whether the factors of COPD status, frequency of hospitalization for COPDAE within one year before the index date, age, menopausal status, CCI score, differentiation, AJCC clinical stage, adjuvant chemotherapy, adjuvant radiotherapy, HR status, Her-2 status, nodal surgery, types of breast surgery, MACE, hypertension, diabetes, COPD, hyperlipidemia, alcohol use, drug abuse, and CKD were potential independent predictors of all-cause mortality. Potential predictors were controlled for in the analysis (Table 1), and all-cause mortality was the primary endpoint in both groups.

Table 1. Characteristics of patients with invasive ductal carcinoma with or without smoking-related COPD before breast surgery after propensity score matching.

\begin{tabular}{|c|c|c|c|c|c|}
\hline \multirow[t]{3}{*}{ Variables } & \multicolumn{2}{|c|}{ Nonsmokers without COPD } & \multicolumn{2}{|c|}{ Smokers with COPD } & \multirow{3}{*}{$p$-Value } \\
\hline & \multicolumn{2}{|c|}{$N=1546$} & \multicolumn{2}{|c|}{$N=773$} & \\
\hline & & $N, \%$ & & $N, \%$ & \\
\hline Age (mean \pm SD) & \multicolumn{2}{|c|}{$(58.37 \pm 12.59)$} & \multicolumn{2}{|c|}{$(58.83 \pm 12.37)$} & 0.404 \\
\hline Age (years) & & & & & 0.467 \\
\hline$\leq 50$ & 396 & $25.61 \%$ & 209 & $27.04 \%$ & \\
\hline $5 \overline{1}-60$ & 444 & $28.72 \%$ & 212 & $27.43 \%$ & \\
\hline $61-70$ & 414 & $26.78 \%$ & 191 & $24.71 \%$ & \\
\hline$>70$ & 292 & $18.89 \%$ & 161 & $20.83 \%$ & \\
\hline CCI score & & & & & 0.310 \\
\hline 0 & 1417 & $91.66 \%$ & 694 & $89.78 \%$ & \\
\hline 1 & 30 & $1.94 \%$ & 20 & $2.59 \%$ & \\
\hline$\geq 2$ & 99 & $6.40 \%$ & 59 & $7.63 \%$ & \\
\hline CCI score (mean $\pm \mathrm{SD})$ & \multicolumn{2}{|c|}{$(0.16 \pm 0.59)$} & \multicolumn{2}{|c|}{$(0.21 \pm 0.70)$} & 0.108 \\
\hline Menopausal status & & & & & 0.320 \\
\hline Postmenopausal & 996 & $64.42 \%$ & 462 & $59.77 \%$ & \\
\hline Premenopausal & 550 & $35.58 \%$ & 311 & $40.23 \%$ & \\
\hline Her2 status & & & & & 0.422 \\
\hline Negative & 1259 & $81.44 \%$ & 618 & $79.95 \%$ & \\
\hline Positive & 287 & $18.56 \%$ & 155 & $20.05 \%$ & \\
\hline Nodal surgery & & & & & 0.891 \\
\hline SLNB & 1082 & $69.99 \%$ & 543 & $70.25 \%$ & \\
\hline ALND & 464 & $30.01 \%$ & 230 & $29.75 \%$ & \\
\hline AJCC clinical stage & & & & & 0.782 \\
\hline I & 801 & $51.81 \%$ & 408 & $52.78 \%$ & \\
\hline II & 376 & $24.32 \%$ & 193 & $24.97 \%$ & \\
\hline III & 369 & $23.87 \%$ & 172 & $22.25 \%$ & \\
\hline Hormone receptor & & & & & 0.792 \\
\hline Negative & 345 & $22.32 \%$ & 177 & $22.90 \%$ & \\
\hline Positive & 1201 & $77.68 \%$ & 596 & $77.10 \%$ & \\
\hline Breast surgery & & & & & 0.726 \\
\hline Total mastectomy & 228 & $14.75 \%$ & 119 & $15.39 \%$ & \\
\hline Breast-conserving surgery & 1318 & $85.25 \%$ & 654 & $84.61 \%$ & \\
\hline Differentiation & & & & & 0.692 \\
\hline $\mathrm{I}$ & 228 & $14.75 \%$ & 119 & $15.39 \%$ & \\
\hline II & 731 & $47.28 \%$ & 351 & $45.41 \%$ & \\
\hline III & 587 & $37.97 \%$ & 303 & $39.20 \%$ & \\
\hline
\end{tabular}


Table 1. Cont.

\begin{tabular}{|c|c|c|c|c|c|}
\hline \multirow[t]{3}{*}{ Variables } & \multicolumn{2}{|c|}{ Nonsmokers without COPD } & \multicolumn{2}{|c|}{ Smokers with COPD } & \multirow{3}{*}{$p$-Value } \\
\hline & \multicolumn{2}{|c|}{$N=1546$} & \multicolumn{2}{|c|}{$N=773$} & \\
\hline & & $N, \%$ & \multicolumn{2}{|r|}{$N, \%$} & \\
\hline \multicolumn{5}{|l|}{ Adjuvant chemotherapy } & 0.177 \\
\hline No & 756 & $48.90 \%$ & 403 & $52.13 \%$ & \\
\hline Yes & 790 & $51.10 \%$ & 370 & $47.87 \%$ & \\
\hline \multicolumn{5}{|l|}{ Adjuvant radiotherapy } & 0.812 \\
\hline No & 228 & $14.75 \%$ & 119 & $15.39 \%$ & \\
\hline Yes & 1318 & $85.25 \%$ & 654 & $84.61 \%$ & \\
\hline \multicolumn{5}{|l|}{ MACE history } & 0.322 \\
\hline No & 1114 & $72.06 \%$ & 541 & $69.99 \%$ & \\
\hline Yes & 432 & $27.94 \%$ & 232 & $30.01 \%$ & \\
\hline \multicolumn{5}{|l|}{ Hyperlipidemia } & 0.566 \\
\hline No & 1138 & $78.16 \%$ & 589 & $76.20 \%$ & \\
\hline Yes & 318 & $21.84 \%$ & 184 & $23.80 \%$ & \\
\hline \multicolumn{5}{|l|}{ Hypertension } & 0.664 \\
\hline No & 965 & $66.28 \%$ & 502 & $64.94 \%$ & \\
\hline Yes & 491 & $33.72 \%$ & 271 & $35.06 \%$ & \\
\hline \multicolumn{5}{|l|}{ Diabetes } & 0.645 \\
\hline No & 1164 & $79.95 \%$ & 610 & $78.91 \%$ & \\
\hline Yes & 292 & $20.05 \%$ & 163 & $21.09 \%$ & \\
\hline \multicolumn{5}{|l|}{ Chronic kidney disease } & 1.000 \\
\hline No & 1441 & $98.97 \%$ & 765 & $98.97 \%$ & \\
\hline Yes & 15 & $1.03 \%$ & 8 & $1.03 \%$ & \\
\hline \multicolumn{5}{|l|}{ Alcohol use } & 0.492 \\
\hline No & 1268 & $82.02 \%$ & 618 & $79.94 \%$ & \\
\hline Yes & 278 & $17.98 \%$ & 155 & $20.06 \%$ & \\
\hline \multicolumn{5}{|l|}{ Drug abuse } & 0.284 \\
\hline No & 1500 & $97.02 \%$ & 743 & $96.12 \%$ & \\
\hline Yes & 46 & $2.98 \%$ & 30 & $3.88 \%$ & \\
\hline \multicolumn{5}{|c|}{$\begin{array}{l}\text { Frequency of hospitalization for COPDAE within } 1 \text { year before breast } \\
\text { surgery }\end{array}$} & $<0.001$ \\
\hline 0 & 1546 & $100.00 \%$ & 702 & $90.82 \%$ & \\
\hline 1 & 0 & $0.00 \%$ & 39 & $5.05 \%$ & \\
\hline$\geq 2$ & 0 & $0.00 \%$ & 32 & $4.14 \%$ & \\
\hline $\begin{array}{l}\text { Follow-up (All-cause } \\
\text { mortality) Years, Median } \\
\text { (IQR, Q1-Q3) }\end{array}$ & & 06) & & & $<0.001$ \\
\hline $\begin{array}{l}\text { Follow-up (Did not die) } \\
\text { Years, Median (IQR, Q1-Q3) }\end{array}$ & & 93) & & & 0.788 \\
\hline
\end{tabular}

IQR, interquartile range; SD, standard deviation; AJCC, American Joint Committee on Cancer; CCI, Charlson comorbidity index; ALND, axillary lymph node dissection; COPD, chronic obstructive pulmonary disease; COPDAE, COPD with acute exacerbation; MACE, major adverse cardiovascular events.

\subsection{Statistics}

Continuous variables are expressed as mean $\pm \mathrm{SD}$. Comparisons among the two groups were conducted using independent $t$-tests for continuous variables and a Chi-square test for categorical variables. The Mann-Whitney $\mathrm{U}$ test is used to compare differences of follow-up time between the two groups. We have used a Gray's test to produce the two $p$ values for adjuvant RT and adjuvant chemotherapy in Table 1. After adjustment for confounders, all analyses were 
performed using SAS version 9.3 (SAS Institute, Cary, NC, USA). In a two-tailed Wald test, $p<0.05$ was considered significant. OS was estimated using the Kaplan-Meier method, and differences among non-COPD, COPD, and hospitalization for COPDAE were determined using the stratified log-rank test to compare survival curves (stratified according to matched sets) [39].

\section{Results}

\subsection{Propensity Score Matching and Study Cohort}

PSM yielded 2319 patients with stage I-III IDC (773 and 1546 in Groups 1 and 2, respectively) eligible for further analysis. Table 1 summarizes their clinicodemographic characteristics. Age, menopausal status, CCI score, differentiation, AJCC clinical stage, adjuvant chemotherapy, adjuvant radiotherapy, HR status, Her-2 status, nodal surgery, types of breast surgery, MACE, hypertension, diabetes, hyperlipidemia, alcohol use, drug abuse, and CKD were similar between the two groups due to PSM. Follow-up duration and hospitalization for COPDAE within one year before breast surgery was inconsistent between the two groups (Table 1).

\subsection{Prognostic Factors of All-Cause Mortality after Multivariate Cox Regression Analyses}

Multivariate Cox regression analysis indicated that hospitalization for COPDAE within one year before the index date, old age, high CCI, advanced AJCC clinical stage, high grade of differentiation, and history of MACE were associated with poor OS (Table 2). No significant differences were observed in menopausal status, adjuvant chemotherapy, adjuvant radiotherapy, HR status, Her-2 status, nodal surgery, types of breast surgery, hypertension, diabetes, COPD, hyperlipidemia, alcohol use, drug abuse, or CKD (Table 2). The adjusted hazard ratio (aHR; 95\% CI) of all-cause mortality for Group 1 compared with Group 2 was 1.04 (0.83-1.22; $p=0.782)$. The aHRs $(95 \% \mathrm{CIs})$ of all-cause mortality for $\geq 1$ hospitalization for COPDAE within one year before breast surgery was $1.51(1.18-2.36 ; p=0.002)$ compared with no COPDAE in patients with stage I-III IDC undergoing breast surgery. Moreover, aHRs (95\% CIs) of allcause mortality for the age groups of 51-60 years, 61-70 years, and $>70$ years; CCI 1 and $\geq 2$; AJCC clinical stage II and III; differentiation grade II and III; and history of MACE were 1.54 (1.12-2.13), 2.32 (1.67-3.21), 4.92 (3.50-6.90); 1.52 (1.24-2.12), 1.85 (1.26-2.70); 1.22 (1.06-1.93) and1.47 (1.13-1.85); 1.03 (1.01-1.47) and 1.08 (1.07-1.35); and 1.31(1.14-2.25) respectively, compared with age $\leq 50$ years; $\mathrm{CCI}=0$; AJCC clinical stage I; differentiation grade I; and no history of MACE, respectively.

Table 2. Cox proportional hazards analysis of all-cause mortality for patients with invasive ductal carcinoma with or without smoking-related COPD before breast surgery.

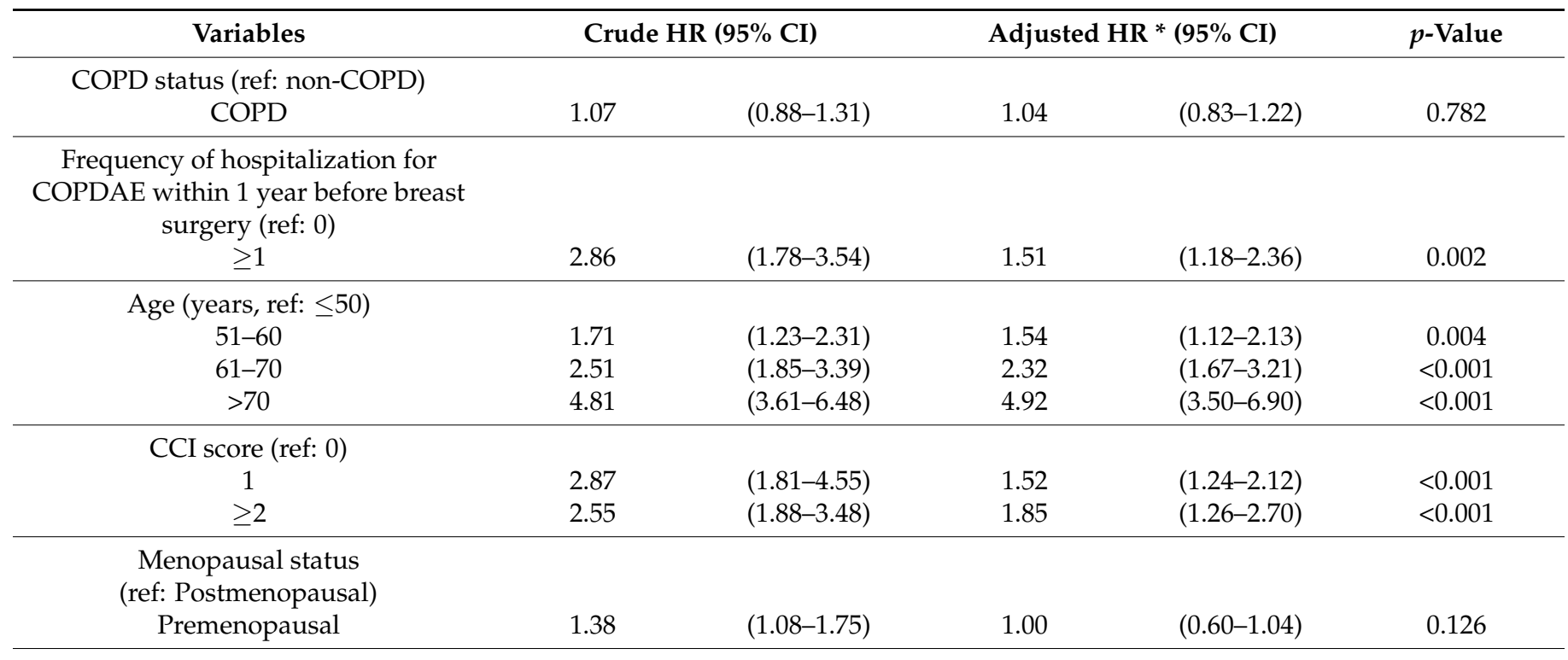


Table 2. Cont.

\begin{tabular}{|c|c|c|c|c|c|}
\hline Variables & \multicolumn{2}{|c|}{ Crude HR $(95 \%$ CI) } & \multicolumn{2}{|c|}{ Adjusted HR * $(95 \% \mathrm{CI})$} & \multirow{2}{*}{$\begin{array}{c}p \text {-Value } \\
0.508\end{array}$} \\
\hline Positive & 1.51 & $(1.18-1.93)$ & 0.89 & $(0.66-1.19)$ & \\
\hline $\begin{array}{l}\text { Breast surgery (ref: Total } \\
\text { mastectomy) } \\
\text { Breast-conserving surgery }\end{array}$ & 1.31 & $(0.86-1.68)$ & 1.11 & $(0.88-1.20)$ & 0.382 \\
\hline $\begin{array}{l}\text { Nodal surgery (ref: SLND) } \\
\text { ALND }\end{array}$ & 1.28 & $(0.50-1.48)$ & 1.18 & $(0.68-1.87)$ & 0.492 \\
\hline $\begin{array}{c}\text { AJCC clinical stage (ref. stage I) } \\
\text { Stage II } \\
\text { Stage III }\end{array}$ & $\begin{array}{l}1.81 \\
2.13\end{array}$ & $\begin{array}{l}(1.23-2.48) \\
(1.60-2.83)\end{array}$ & $\begin{array}{l}1.22 \\
1.47\end{array}$ & $\begin{array}{l}(1.06-1.93) \\
(1.13-1.85)\end{array}$ & $\begin{array}{l}0.003 \\
0.008\end{array}$ \\
\hline $\begin{array}{c}\text { Hormone receptor (ref. Negative) } \\
\text { Positive }\end{array}$ & 0.92 & $(0.81-1.40)$ & 0.90 & $(0.87-1.37)$ & 0.337 \\
\hline $\begin{array}{c}\text { Differentiation (ref: Grade I) } \\
\text { Grade II } \\
\text { Grade III }\end{array}$ & $\begin{array}{l}1.08 \\
1.12\end{array}$ & $\begin{array}{l}(1.02-1.36) \\
(1.04-1.38)\end{array}$ & $\begin{array}{l}1.03 \\
1.08\end{array}$ & $\begin{array}{l}(1.01-1.47) \\
(1.07-1.35)\end{array}$ & $\begin{array}{l}0.044 \\
0.013\end{array}$ \\
\hline $\begin{array}{c}\text { Adjuvant chemotherapy (ref: No) } \\
\text { Yes }\end{array}$ & 0.73 & $(0.43-1.10)$ & 0.83 & $(0.72-1.06)$ & 0.361 \\
\hline $\begin{array}{c}\text { Adjuvant radiotherapy (ref: No) } \\
\text { Yes }\end{array}$ & 0.77 & $(0.46-1.13)$ & 0.70 & $(0.52-1.09)$ & 0.304 \\
\hline $\begin{array}{l}\text { MACE history (ref: No) } \\
\text { Yes }\end{array}$ & 1.16 & $(1.01-2.57)$ & 1.31 & $(1.14-2.25)$ & 0.005 \\
\hline $\begin{array}{c}\text { Hyperlipidemia (ref: No) } \\
\text { Yes }\end{array}$ & 1.65 & $(1.01-2.24)$ & 0.93 & $(0.61-1.51)$ & 0.798 \\
\hline $\begin{array}{l}\text { Hypertension (ref: No) } \\
\text { Yes }\end{array}$ & 1.66 & $(1.13-2.45)$ & 1.13 & $(0.71-1.79)$ & 0.521 \\
\hline $\begin{array}{c}\text { Diabetes (ref: No) } \\
\text { Yes }\end{array}$ & 1.90 & $(1.35-2.66)$ & 1.43 & $(0.97-2.11)$ & 0.061 \\
\hline $\begin{array}{c}\text { Chronic kidney disease (ref: No) } \\
\text { Yes }\end{array}$ & 1.28 & $(0.88-1.84)$ & 1.01 & $(0.48-1.16)$ & 0.174 \\
\hline $\begin{array}{c}\text { Alcohol use (ref: No) } \\
\text { Yes }\end{array}$ & 1.44 & $(0.98-2.13)$ & 0.98 & $(0.69-1.56)$ & 0.452 \\
\hline $\begin{array}{c}\text { Drug abuse (ref: No) } \\
\text { Yes }\end{array}$ & 1.39 & $(0.71-2.49)$ & 0.90 & $(0.65-1.63)$ & 0.833 \\
\hline
\end{tabular}

AJCC, American Joint Committee on Cancer; CCI, Charlson comorbidity index; ALND, axillary lymph node dissection; COPD, chronic obstructive pulmonary disease; COPDAE, COPD with acute exacerbation; MACE, major adverse cardiovascular events; ref, reference group. * All covariates mentioned in Table 2 were adjusted.

\subsection{Kaplan-Meier OS among Non-COPD, COPD, and Hospitalization for COPDAE}

Figure 1 presents the Kaplan-Meier survival curves for the two groups. The OS was not significantly different between the two groups $(p=0.983)$. The OS of patients with IDC with $\geq 1$ hospitalization for COPDAE within one year before breast surgery was poorer than that for those with 0 hospitalizations for COPDAE $(p<0.001)$ (Figure 2). 


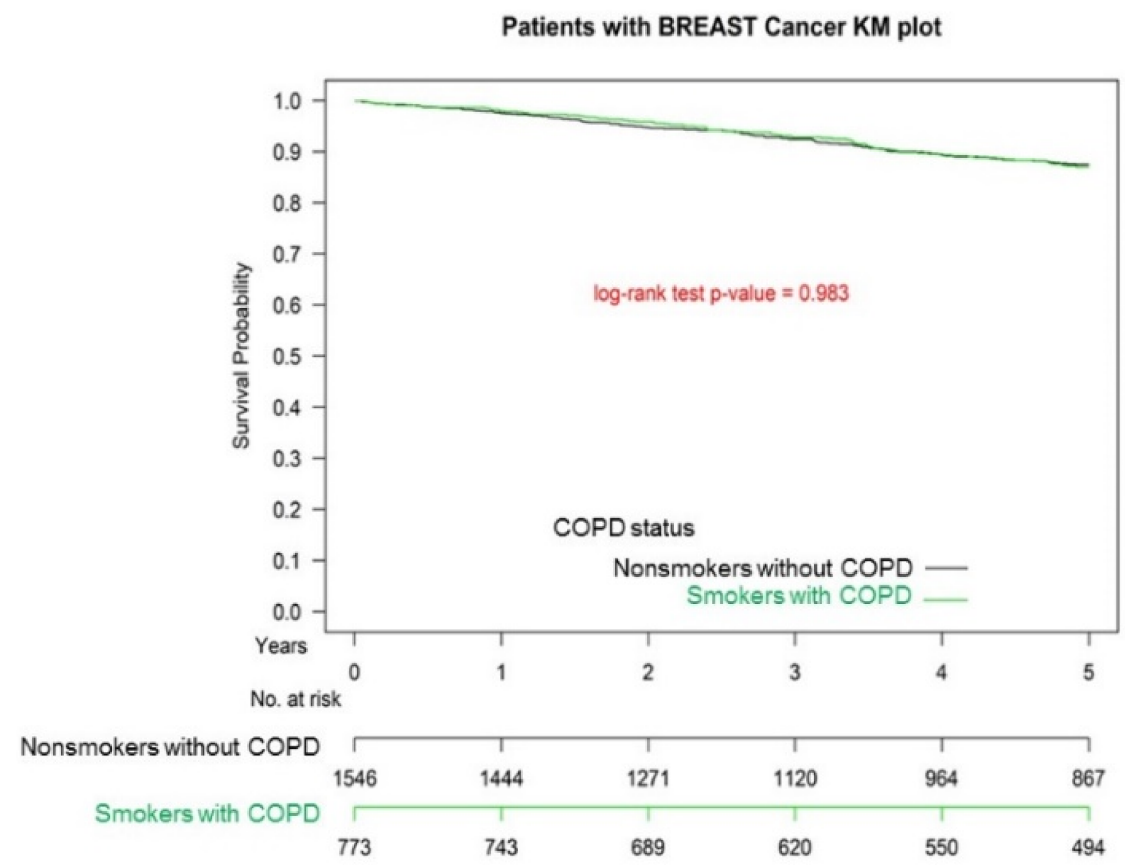

Figure 1. Kaplan-Meier survival curves of patients with invasive ductal carcinoma with or without smoking-related chronic obstructive pulmonary disease (COPD) before breast surgery.

Patients with BREAST Cancer KM plot

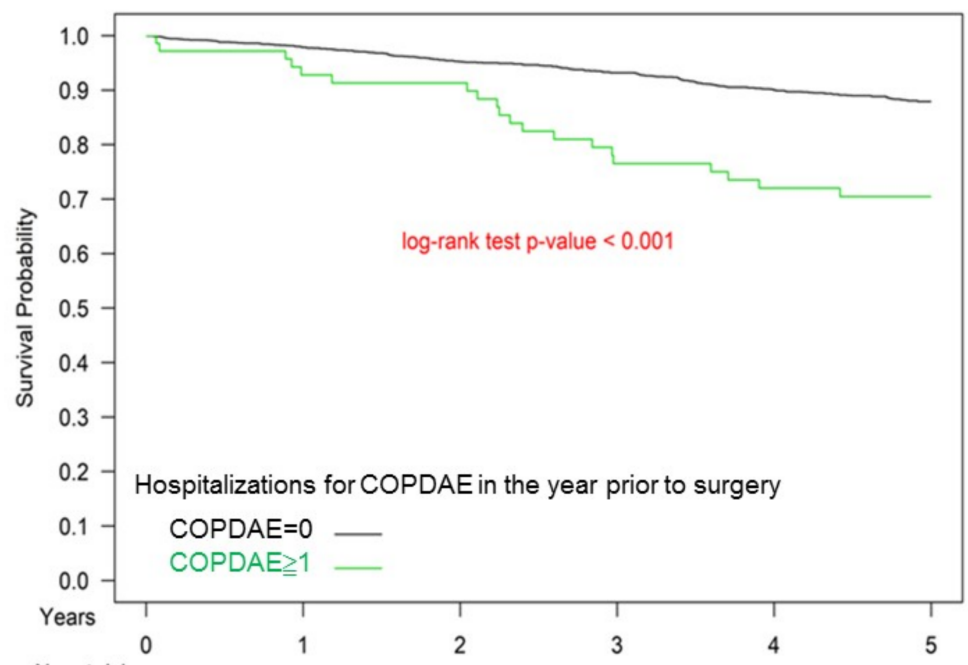

No. at risk

\begin{tabular}{|c|c|c|c|c|c|c|}
\hline COPDAE $=0$ & г & $T$ & $T$ & $T$ & T & $\neg$ \\
\hline & 2248 & 2119 & 1898 & 1684 & 1463 & 1316 \\
\hline COPDAE $\geqq 1$ & $\Gamma$ & $T$ & $T$ & $T$ & $T$ & ᄀ \\
\hline & 71 & 68 & 62 & 56 & 51 & 45 \\
\hline
\end{tabular}

Figure 2. Kaplan-Meier survival curves of patients with invasive ductal carcinoma with frequency of hospitalization for COPDAE within 1 year before breast surgery. COPDAE, chronic obstruction pulmonary disease with acute exacerbation.

\section{Discussion}

The mechanism is largely unclear by which COPD increases cancer risk [40]. Smoking is a common shared risk factor for COPD and solid organ cancers (including breast cancer) [40]. However, even after adjusting for smoking, a significant relationship between COPD and cancer was observed [40]. Among patients with breast cancer, comorbidities in general and specifically cardiovascular diseases, COPD, diabetes, and venous thromboembolism negatively affect OS [41]. 
Thus, smoking related COPDAE might contribute to poor OS in patients with breast cancer receiving breast surgery, although no study has analyzed this. The severity of cigarette smokingrelated COPD might be proportional to the severity of poor pulmonary function [1-4] or poor cardiac function [5] attributable to the higher mortality due to the progression of toxicity by treatments of IDC. Ours is the first study to evaluate whether the severity of smoking-related COPD is a significant prognostic factor of OS in the patients with IDC receiving standard treatments.

Because of PSM, all potential covariates associated with the OS of breast cancer patients receiving treatments were well-matched between the two groups. Our study is the first head-to-head PSM study to estimate the severity of current smoking-related COPD or COPDAE for patients with IDC undergoing breast surgery and standard adjuvant treatments based on NCCN guidelines [11].

Our data indicated no significant association of cigarette smoking-related COPD and OS for women with IDC receiving standard treatments. No study has analyzed smoking-related COPD as a risk factor for all-cause mortality in patients with breast cancer receiving treatments, even though cigarette smoking is significantly associated with a poor prognosis in women diagnosed with breast cancer [42]. Our study is the first study to show that current smoker-related COPD was not a significant prognostic factor for women with IDC receiving standard treatments. We found that hospitalization for COPDAE within one year before breast treatment was an independent prognostic factor of OS. The severity of smoking-related COPD, evident in occurrences such as hospitalization for COPDAE (the same as the Global Initiative for Chronic Obstructive Lung Disease [GOLD] Classification 3-4) [43], was a significant independent prognostic factor of mortality for women with IDC receiving standard treatments. This may be because severe COPDAE with poor pulmonary or cardiac function worsened the OS in patients with IDC receiving standard treatments, probably due to radiation-induced intolerable lung injury (RILI) or treatment-induced cardiotoxicity [12-20].

Preexisting COPD and female sex are associated with an increased risk of radiation pneumonitis in patients with breast cancer undergoing radiotherapy $[19,20]$. In addition, not only COPD-related RILI but also patient-related factors may increase the risk of radiation-induced cardiotoxicity, including coronary heart disease, one of the MACE [12,13]. Preexisting cardiovascular disease (one of the MACE) may increase the radiation induced cardiac toxicity (RICT) $[12,13]$. In addition, cancer patients receiving chemotherapy have an increased risk of cardiovascular complications, and the risk is even greater with a history of heart disease $[44,45]$. Anthracycline and anthracycline-like agents [14-18,46] and HER2targeting agents, such as trastuzumab [47,48] and fluoropyrimidines [44], are anticancer agents that are well known to be associated with cardiac toxicity. Concomitant chronic cardiac disorders such as MACE are frequent in patients with COPD [49]. Risk factors for anthracycline cardiac toxicity include female sex, COPD, and MACE [14-18,46,49]. Therefore, COPD or MACE may lead to more severe cardiotoxicity after systemic therapy in patients with breast cancer [14-18,44,46-49]. In our study, a history of MACE associated with higher risk of all-cause mortality after multivariate analysis echoes the above findings (Table 2) [14-18,46]. Thus, hospitalization for COPDAE within one year before standard treatments for IDC might indicate that poorer pulmonary (RILI) or poor cardiac function (RICT or chemotherapy induced cardiac toxicity) [1-5] contributed to worse survival compared with those without COPDAE (Table 2).

In our study, MACE and preexisting COPDAE, but not hypertension, diabetes, hyperlipidemia, COPD, alcohol use, drug abuse, or CKD, were significant prognostic factors of all-cause mortality in patients with IDC receiving standard treatments (Table 2). In a previous study, preoperative MACE (adjusted odds ratio, 1.21; 95\% CI, 1.14-1.29) were found to be a prognostic marker for perioperative 30-day morbidity and mortality for cancer patients [50]. In our study, MACE were an independent risk factor for all-cause mortality for patients with IDC receiving standard treatments, in accord with other studies [12-18]. MACE seem to be more predictive for OS of patients with IDC receiving standard treatments than hypertension, diabetes, COPD, hyperlipidemia, alcohol use, drug abuse, or 
CKD (Table 2). The other poor prognostic factors of all-cause mortality for patients with IDC undergoing breast surgery and adjuvant treatments according to NCCN guidelines were old age, high CCI, high grade differentiation, and advanced clinical stages (Table 2), in accordance with previous studies [51-55].

Neoadjuvant chemotherapy is associated with high rates of clinical response and a greater likelihood of facilitating cosmetically acceptable surgery [22,24,56]. For example, patients who were not candidates for breast conservation may become eligible after neoadjuvant chemotherapy [22,24,56]. Most patients with early stage (AJCC stage I-II) breast cancer receiving breast surgery would not need neoadjuvant chemotherapy in our study, compatible with other studies $[22,24,56]$. Thus, fewer patients received neoadjuvant chemotherapy in our study, because there were more than $75 \%$ stage I-II early stage breast cancers in the current study (Table 1). Additionally, various regimens and different courses of neoadjuvant chemotherapy would cause too many covariates in our analysis. Moreover, the response rates (complete response, partial response, stationary disease, and progression of disease) of neoadjuvant chemotherapy are strongly associated with survival for women with breast cancer receiving neoadjuvant chemotherapy $[23,25]$. Therefore, if we consider including neoadjuvant chemotherapy for fewer patients with early breast IDC, we need to consider additional covariates including response rate (complete response, partial response, stationary disease, and progression of disease), regimens of chemotherapy, and courses (four, six, or eight courses) of neoadjuvant chemotherapy in our study [22-25,56]. However, too many covariates in a multivariable model may cause the problem of overfitting [57], especially in a small sample size for neoadjuvant chemotherapy, as in our study. In addition, a regression model containing too many variables would result in overspecified bias [58].

The strength of our study was that it was the first and largest cohort study to estimate the survival outcomes of current smoking-related COPD compared with nonsmokers without COPD among patients with IDC undergoing breast surgery and adjuvant treatments based on NCCN guidelines [11]. PSM led to comparable covariates between groups, and no selection bias was noted (Table 1). No prior study has estimated the impact of COPD and hospitalization for COPDAE in breast cancer patients receiving standard treatments, and all of the prognostic factors were evaluated. In our study, the poor prognostic factors of OS in these patients with breast cancer were similar, such as CCI $\geq 1$, moderate to poor differentiation, advanced clinical stages II-III, and old age (Table 2), and were in accord with previous studies [51-55]. Until now, there has been no evidence for proving the risk of all-cause death for COPDAE before treatment of breast cancer, and resulting worse survival. This is the first study to demonstrate with real world data that COPD was not associated with overall survival; 1+ hospitalizations for COPDAE in the year prior to surgery was associated with an increased risk of death. Because 1+ hospitalizations for COPDAE in the year prior to surgery was associated with an increased risk of death, well-controlled COPD disease prevention from COPDAE is valuable for breast cancer survival in future clinical practice. In addition, 1+ hospitalizations for COPDAE in the year prior to surgery was associated with an increased risk of death, and should be considered in prospective clinical trials for breast cancer research.

There are some limitations in our study. First, all IDC patients are from Asian populations; therefore, our results should be carefully extrapolated to non-Asian populations. However, there is no evidence that there is a difference in the oncology results of IDC patients receiving standard treatment between Asian and non-Asian populations. Second, the diagnosis of all comorbidities is based on the ICD-10-CM code. The Taiwan Cancer Registry Administration randomly reviewed medical records and interviewed patients to verify the accuracy of the diagnosis. If improper behavior or discrepancies are found, hospitals with abnormal charges or practices will be audited and severely punished. However, in order to obtain critical information about population specificity and disease occurrence, large-scale randomized trials must be conducted to compare carefully selected patients receiving appropriate treatment. Finally, the TCRD does not contain information on socioeconomic status, body mass index, or eating habits, all of which may be risk factors for death in IDC 
patients. However, given the magnitude and statistical significance of the effects observed in this study, these limitations are unlikely to affect the conclusions.

\section{Conclusions}

No association of survival outcomes was observed in women with IDC undergoing breast surgery who had current smoking-related COPD or who were nonsmokers but had COPD. Hospitalization for COPDAE within one year before breast surgery was found to be an independent risk factor for OS for women with IDC receiving standard treatments.

Author Contributions: Conception and Design: J.-Q.Z., K.-C.C., W.-C.L., S.-Y.W. Collection and Assembly of Data: J.-Q.Z., K.-C.C., W.-C.L. Data Analysis and Interpretation: J.-Q.Z., K.-C.C., T.-M.C., W.-C.L., S.-Y.W. Administrative Support: S.-Y.W. Manuscript Writing: J.-Q.Z., K.-C.C., W.-C.L., S.-Y.W. Final Approval of Manuscript: All authors. All authors have read and agreed to the published version of the manuscript.

Funding: Lo-Hsu Medical Foundation, LotungPoh-Ai Hospital, supports Szu-Yuan Wu's work (Funding Number: 10908, 10909, 11001, 11002, 11003, 11006, and 11013). Taipei Medical UniversityWan Fang Hospital (Funding Number: 107TMU-WFH-08) supports Tsai-Mu Cheng's work.

Institutional Review Board Statement: The study protocols were reviewed and approved by the Institutional Review Board of the Tzu-Chi Medical Foundation (IRB109-015-B).

Informed Consent Statement: Patient consent was waived because data files are de-identified by scrambling the identification codes of both patients and medical facilities and sent to the National Health Research Institutes to form the original files of NHIRD. We used data from the National Health Insurance Research Database (NHIRD). The authors confirm that, for approved reasons, some access restrictions apply to the data underlying the findings. The data utilized in this study cannot be made available in the manuscript, the supplementary files, or in a public repository due to the "Personal Information Protection Act" executed by Taiwan's government, starting from 2012. Requests for data can be sent as a formal proposal to obtain approval from the ethics review committee of the appropriate governmental department in Taiwan. Specifically, links regarding contact info for which data requests may be sent to are as follows: http://nhird.nhri.org.tw/en/Data_Subsets.html\#S3 and http://nhis.nhri.org.tw/point.html.

Data Availability Statement: The data sets supporting the study conclusions are included in this manuscript.

Conflicts of Interest: The authors have no potential conflict of interest to declare.

\section{Abbreviations}

COPD Chronic Obstructive Pulmonary Disease

IDC Invasive Ductal Carcinoma

COPDAE COPD with Acute Exacerbation

aHR Adjusted Hazard Ratio

CI Confidence Interval

MACE Major Adverse Cardiac Events

AJCC American Joint Committee on Cancer

RILI Radiation-Induced Lung Injury

RP Radiation Pneumonitis

TCRD Taiwan Cancer Registry Database

PSM Propensity Score Matching

SD Standard Deviation

AJCC American Joint Committee on Cancer

HR Hormone Receptor

Her-2 Human Epidermal Growth Factor Receptor-2

OS Overall Survival

SLNB Sentinel Lymph Node Biopsy

ALND Axillary Lymph Node Dissection

CKD Chronic Kidney Disease 


$\begin{array}{ll}\text { CCI } & \text { Charlson Comorbidity Index } \\ \text { ICD-10-CM } & \text { International Classification of Diseases, 10th Revision, Clinical Modification } \\ \text { NCCN } & \text { National Comprehensive Cancer Network } \\ \text { GOLD } & \text { Global Initiative for Chronic Obstructive Lung Disease }\end{array}$

\section{References}

1. Bhatt, S.P.; Dransfield, M.T. Chronic obstructive pulmonary disease and cardiovascular disease. Transl. Res. 2013, 162, 237-251. [CrossRef] [PubMed]

2. Laratta, C.R.; van Eeden, S. Acute exacerbation of chronic obstructive pulmonary disease: Cardiovascular links. Biomed. Res. Int. 2014, 2014, 528789. [CrossRef] [PubMed]

3. Ghoorah, K.; De Soyza, A.; Kunadian, V. Increased cardiovascular risk in patients with chronic obstructive pulmonary disease and the potential mechanisms linking the two conditions: A review. Cardiol. Rev. 2013, 21, 196-202. [CrossRef] [PubMed]

4. Lee, P.N. The effect of reducing the number of cigarettes smoked on risk of lung cancer, COPD, cardiovascular disease and FEV(1)—A Review. Regul. Toxicol. Pharmacol. 2013, 67, 372-381. [CrossRef]

5. Reilev, M.; Pottegard, A.; Lykkegaard, J.; Sondergaard, J.; Ingebrigtsen, T.S.; Hallas, J. Increased risk of major adverse cardiac events following the onset of acute exacerbations of COPD. Respirology 2019, 24, 1183-1190. [CrossRef]

6. Gram, I.T.; Park, S.Y.; Kolonel, L.N.; Maskarinec, G.; Wilkens, L.R.; Henderson, B.E.; Le Marchand, L. Smoking and Risk of Breast Cancer in a Racially/Ethnically Diverse Population of Mainly Women Who Do Not Drink Alcohol: The MEC Study. Am. J. Epidemiol. 2015, 182, 917-925. [CrossRef]

7. Gaudet, M.M.; Carter, B.D.; Brinton, L.A.; Falk, R.T.; Gram, I.T.; Luo, J.; Milne, R.L.; Nyante, S.J.; Weiderpass, E.; Beane Freeman, L.E.; et al. Pooled analysis of active cigarette smoking and invasive breast cancer risk in 14 cohort studies. Int. J. Epidemiol. 2017, 46, 881-893. [CrossRef]

8. Macacu, A.; Autier, P.; Boniol, M.; Boyle, P. Active and passive smoking and risk of breast cancer: A meta-analysis. Breast Cancer Res. Treat. 2015, 154, 213-224. [CrossRef]

9. Gaudet, M.M.; Gapstur, S.M.; Sun, J.; Diver, W.R.; Hannan, L.M.; Thun, M.J. Active smoking and breast cancer risk: Original cohort data and meta-analysis. J. Natl. Cancer Inst. 2013, 105, 515-525. [CrossRef]

10. Johnson, K.C.; Miller, A.B.; Collishaw, N.E.; Palmer, J.R.; Hammond, S.K.; Salmon, A.G.; Cantor, K.P.; Miller, M.D.; Boyd, N.F.; Millar, J.; et al. Active smoking and secondhand smoke increase breast cancer risk: The report of the Canadian Expert Panel on Tobacco Smoke and Breast Cancer Risk (2009). Tob. Control. 2011, 20, e2. [CrossRef]

11. NCCN Clinical Practice Guidelines in Oncology. Available online: http://www.nccn.org/professionals/physician_gls/f_ guidelines.asp (accessed on 28 June 2021)

12. Darby, S.C.; Ewertz, M.; McGale, P.; Bennet, A.M.; Blom-Goldman, U.; Bronnum, D.; Correa, C.; Cutter, D.; Gagliardi, G.; Gigante, B.; et al. Risk of ischemic heart disease in women after radiotherapy for breast cancer. N. Engl. J. Med. 2013, 368, 987-998. [CrossRef]

13. Hooning, M.J.; Botma, A.; Aleman, B.M.; Baaijens, M.H.; Bartelink, H.; Klijn, J.G.; Taylor, C.W.; van Leeuwen, F.E. Long-term risk of cardiovascular disease in 10-year survivors of breast cancer. J. Natl. Cancer Inst. 2007, 99, 365-375. [CrossRef]

14. Qin, A.; Thompson, C.L.; Silverman, P. Predictors of late-onset heart failure in breast cancer patients treated with doxorubicin. J. Cancer Surviv. 2015, 9, 252-259. [CrossRef]

15. Plana, J.C.; Galderisi, M.; Barac, A.; Ewer, M.S.; Ky, B.; Scherrer-Crosbie, M.; Ganame, J.; Sebag, I.A.; Agler, D.A.; Badano, L.P.; et al. Expert consensus for multimodality imaging evaluation of adult patients during and after cancer therapy: A report from the American Society of Echocardiography and the European Association of Cardiovascular Imaging. J. Am. Soc. Echocardiogr. Off. Publ. Am. Soc. Echocardiogr. 2014, 27, 911-939. [CrossRef]

16. Drafts, B.C.; Twomley, K.M.; D'Agostino, R., Jr.; Lawrence, J.; Avis, N.; Ellis, L.R.; Thohan, V.; Jordan, J.; Melin, S.A.; Torti, F.M.; et al. Low to moderate dose anthracycline-based chemotherapy is associated with early noninvasive imaging evidence of subclinical cardiovascular disease. JACC Cardiovasc. Imaging 2013, 6, 877-885. [CrossRef]

17. Swain, S.M.; Whaley, F.S.; Ewer, M.S. Congestive heart failure in patients treated with doxorubicin: A retrospective analysis of three trials. Cancer 2003, 97, 2869-2879. [CrossRef]

18. Doroshow, J.H. Doxorubicin-induced cardiac toxicity. N. Engl. J. Med. 1991, 324, 843-845. [CrossRef]

19. Rancati, T.; Ceresoli, G.L.; Gagliardi, G.; Schipani, S.; Cattaneo, G.M. Factors predicting radiation pneumonitis in lung cancer patients: A retrospective study. Radiother. Oncol. 2003, 67, 275-283. [CrossRef]

20. Robnett, T.J.; Machtay, M.; Vines, E.F.; McKenna, M.G.; Algazy, K.M.; McKenna, W.G. Factors predicting severe radiation pneumonitis in patients receiving definitive chemoradiation for lung cancer. Int. J. Radiat. Oncol. Biol. Phys. 2000, 48, 89-94. [CrossRef]

21. Yu, J.M.; Hsieh, M.C.; Qin, L.; Zhang, J.; Wu, S.Y. Metformin reduces radiation-induced cardiac toxicity risk in patients having breast cancer. Am. J. Cancer Res. 2019, 9, 1017-1026.

22. Zhang, J.; Lu, C.Y.; Chen, C.H.; Chen, H.M.; Wu, S.Y. Effect of pathologic stages on postmastectomy radiation therapy in breast cancer receiving neoadjuvant chemotherapy and total mastectomy: A Cancer Database Analysis. Breast 2020, 54, 70-78. [CrossRef]

23. Zhang, J.; Lu, C.Y.; Chen, H.M.; Wu, S.Y. Pathologic response rates for breast cancer stages as a predictor of outcomes in patients receiving neoadjuvant chemotherapy followed by breast-conserving surgery. Surg. Oncol. 2020, 36, 91-98. [CrossRef] 
24. Zhang, J.; Lu, C.Y.; Qin, L.; Chen, H.M.; Wu, S.Y. Breast-conserving surgery with or without irradiation in women with invasive ductal carcinoma of the breast receiving preoperative systemic therapy: A cohort study. Breast 2020, 54, 139-147. [CrossRef]

25. Zhang, J.; Sun, M.; Chang, E.; Lu, C.Y.; Chen, H.M.; Wu, S.Y. Pathologic response as predictor of recurrence, metastasis, and survival in breast cancer patients receiving neoadjuvant chemotherapy and total mastectomy. Am. J. Cancer Res. 2020, 10, 3415-3427. [CrossRef]

26. Zhang, J.Q.; Lu, C.Y.; Qin, L.; Chen, H.M.; Wu, S.Y. Outcome of post-mastectomy radiotherapy after primary systemic treatment in patients with different clinical tumor and nodal stages of breast cancer: A cohort study. Am. J. Cancer Res. 2020, 10, 2185-2198.

27. Hammond, M.E.; Hayes, D.F.; Dowsett, M.; Allred, D.C.; Hagerty, K.L.; Badve, S.; Fitzgibbons, P.L.; Francis, G.; Goldstein, N.S.; Hayes, M.; et al. American Society of Clinical Oncology/College of American Pathologists guideline recommendations for immunohistochemical testing of estrogen and progesterone receptors in breast cancer. J. Clin. Oncol. Off. J. Am. Soc. Clin. Oncol. 2010, 28, 2784-2795. [CrossRef]

28. Fehrenbacher, L.; Cecchini, R.S.; Geyer, C.E., Jr.; Rastogi, P.; Costantino, J.P.; Atkins, J.N.; Crown, J.P.; Polikoff, J.; Boileau, J.F.; Provencher, L.; et al. NSABP B-47/NRG Oncology Phase III Randomized Trial Comparing Adjuvant Chemotherapy with or Without Trastuzumab in High-Risk Invasive Breast Cancer Negative for HER2 by FISH and With IHC1+ or 2. J. Clin. Oncol. Off. J. Am. Soc. Clin. Oncol. 2020, 38, 444-453. [CrossRef]

29. Bahreini, F.; Soltanian, A.R.; Mehdipour, P. A meta-analysis on concordance between immunohistochemistry (IHC) and fluorescence in situ hybridization (FISH) to detect HER2 gene overexpression in breast cancer. Breast Cancer 2015, 22, 615-625. [CrossRef]

30. Charlson, M.; Szatrowski, T.P.; Peterson, J.; Gold, J. Validation of a combined comorbidity index. J. Clin. Epidemiol. 1994, 47, 1245-1251. [CrossRef]

31. Chen, J.H.; Yen, Y.C.; Yang, H.C.; Liu, S.H.; Yuan, S.P.; Wu, L.L.; Lee, F.P.; Lin, K.C.; Lai, M.T.; Wu, C.C.; et al. Curative-Intent Aggressive Treatment Improves Survival in Elderly Patients with Locally Advanced Head and Neck Squamous Cell Carcinoma and High Comorbidity Index. Medicine 2016, 95, e3268. [CrossRef] [PubMed]

32. Bonora, B.M.; Avogaro, A.; Fadini, G.P. Extraglycemic Effects of SGLT2 Inhibitors: A Review of the Evidence. Diabetes Metab. Syndr. Obes. 2020, 13, 161-174. [CrossRef] [PubMed]

33. Chong, W.H.; Yanoff, L.B.; Andraca-Carrera, E.; Thanh Hai, M. Assessing the Safety of Glucose-Lowering Drugs-A New Focus for the FDA. N. Engl. J. Med. 2020, 383, 1199-1202. [CrossRef] [PubMed]

34. de Jong, M.; van der Worp, H.B.; van der Graaf, Y.; Visseren, F.L.J.; Westerink, J. Pioglitazone and the secondary prevention of cardiovascular disease. A meta-analysis of randomized-controlled trials. Cardiovasc. Diabetol. 2017, 16, 134. [CrossRef] [PubMed]

35. Arnott, C.; Li, Q.; Kang, A.; Neuen, B.L.; Bompoint, S.; Lam, C.S.P.; Rodgers, A.; Mahaffey, K.W.; Cannon, C.P.; Perkovic, V.; et al. Sodium-Glucose Cotransporter 2 Inhibition for the Prevention of Cardiovascular Events in Patients with Type 2 Diabetes Mellitus: A Systematic Review and Meta-Analysis. J. Am. Heart Assoc. 2020, 9, e014908. [CrossRef]

36. Ramchand, J.; Patel, S.K.; Srivastava, P.M.; Farouque, O.; Burrell, L.M. Elevated plasma angiotensin converting enzyme 2 activity is an independent predictor of major adverse cardiac events in patients with obstructive coronary artery disease. PLoS ONE 2018, 13, e0198144. [CrossRef]

37. Austin, P.C. Optimal caliper widths for propensity-score matching when estimating differences in means and differences in proportions in observational studies. Pharm. Stat. 2011, 10, 150-161. [CrossRef]

38. Austin, P.C. The performance of different propensity score methods for estimating marginal hazard ratios. Stat. Med. 2013, 32, 2837-2849. [CrossRef]

39. Austin, P.C. The use of propensity score methods with survival or time-to-event outcomes: Reporting measures of effect similar to those used in randomized experiments. Stat. Med. 2014, 33, 1242-1258. [CrossRef]

40. van Gestel, Y.R.; Hoeks, S.E.; Sin, D.D.; Huzeir, V.; Stam, H.; Mertens, F.W.; van Domburg, R.T.; Bax, J.J.; Poldermans, D. COPD and cancer mortality: The influence of statins. Thorax 2009, 64, 963-967. [CrossRef]

41. Janssen-Heijnen, M.L.; Maas, H.A.; Houterman, S.; Lemmens, V.E.; Rutten, H.J.; Coebergh, J.W. Comorbidity in older surgical cancer patients: Influence on patient care and outcome. Eur. J. Cancer 2007, 43, 2179-2193. [CrossRef]

42. Pierce, J.P.; Patterson, R.E.; Senger, C.M.; Flatt, S.W.; Caan, B.J.; Natarajan, L.; Nechuta, S.J.; Poole, E.M.; Shu, X.O.; Chen, W.Y. Lifetime cigarette smoking and breast cancer prognosis in the after Breast Cancer Pooling Project. J. Natl. Cancer Inst. 2014, 106, djt359. [CrossRef]

43. Global Initiative for Chronic Obstructive Lung Disease. Global Strategy for the Diagnosis, Management, and Prevention of Chronic Obstructive Pulmonary Disease (2018 Report); Global Initiative for Chronic Obstructive Lung Disease, Inc.: Fontana, WI, USA, 2018.

44. Anand, A.J. Fluorouracil cardiotoxicity. Ann. Pharmacother. 1994, 28, 374-378. [CrossRef]

45. Akhtar, S.S.; Salim, K.P.; Bano, Z.A. Symptomatic cardiotoxicity with high-dose 5-fluorouracil infusion: A prospective study. Oncology 1993, 50, 441-444. [CrossRef]

46. Lipshultz, S.E.; Colan, S.D.; Gelber, R.D.; Perez-Atayde, A.R.; Sallan, S.E.; Sanders, S.P. Late cardiac effects of doxorubicin therapy for acute lymphoblastic leukemia in childhood. N. Engl. J. Med. 1991, 324, 808-815. [CrossRef]

47. Perez, E.A.; Rodeheffer, R. Clinical cardiac tolerability of trastuzumab. J. Clin. Oncol. Off. J. Am. Soc. Clin. Oncol. 2004, 22, 322-329. [CrossRef]

48. Keefe, D.L. Trastuzumab-associated cardiotoxicity. Cancer 2002, 95, 1592-1600. [CrossRef] 
49. Roversi, S.; Fabbri, L.M.; Sin, D.D.; Hawkins, N.M.; Agusti, A. Chronic Obstructive Pulmonary Disease and Cardiac Diseases. An Urgent Need for Integrated Care. Am. J. Respir. Crit. Care Med. 2016, 194, 1319-1336. [CrossRef]

50. Leung, A.A.; McAlister, F.A.; Rogers, S.O., Jr.; Pazo, V.; Wright, A.; Bates, D.W. Preoperative hyponatremia and perioperative complications. Arch. Intern. Med. 2012, 172, 1474-1481. [CrossRef]

51. Yoo, S.; Lee, H.B.; Han, W.; Noh, D.Y.; Park, S.K.; Kim, W.H.; Kim, J.T. Total Intravenous Anesthesia versus Inhalation Anesthesia for Breast Cancer Surgery: A Retrospective Cohort Study. Anesthesiology 2019, 130, 31-40. [CrossRef]

52. Oh, T.K.; Kim, H.H.; Jeon, Y.T. Retrospective analysis of 1-year mortality after gastric cancer surgery: Total intravenous anesthesia versus volatile anesthesia. Acta Anaesthesiol. Scand. 2019, 63, 1169-1177. [CrossRef]

53. Lee, J.H.; Kang, S.H.; Kim, Y.; Kim, H.A.; Kim, B.S. Effects of propofol-based total intravenous anesthesia on recurrence and overall survival in patients after modified radical mastectomy: A retrospective study. Korean J. Anesthesiol. 2016, 69, 126-132. [CrossRef]

54. Enlund, M.; Berglund, A.; Ahlstrand, R.; Wallden, J.; Lundberg, J.; Warnberg, F.; Ekman, A.; Sjoblom Widfeldt, N.; Enlund, A.; Bergkvist, L. Survival after primary breast cancer surgery following propofol or sevoflurane general anesthesia-A retrospective, multicenter, database analysis of 6305 Swedish patients. Acta Anaesthesiol. Scand. 2020, 64, 1048-1054. [CrossRef]

55. Makito, K.; Matsui, H.; Fushimi, K.; Yasunaga, H. Volatile versus Total Intravenous Anesthesia for Cancer Prognosis in Patients Having Digestive Cancer Surgery. Anesthesiology 2020, 133, 764-773. [CrossRef]

56. Zhang, J.; Lu, C.Y.; Chen, H.M.; Wu, S.Y. Neoadjuvant Chemotherapy or Endocrine Therapy for Invasive Ductal Carcinoma of the Breast with High Hormone Receptor Positivity and Human Epidermal Growth Factor Receptor 2 Negativity. JAMA Netw. Open 2021, 4, e211785. [CrossRef]

57. Zhang, Z. Too much covariates in a multivariable model may cause the problem of overfitting. J. Thorac. Dis. 2014, 6, E196-E197. [CrossRef]

58. Elston, D.A.; Proe, M.F. Smoothing Regression Coefficients in an Overspecified Regression Model with Interrelated Explanatory Variables. J. R. Stat. Soc. Ser. C (Appl. Stat.) 1995, 44, 395-406. [CrossRef] 